\title{
Zero divisors for matrices over commutative semirings
}

\author{
Asmaa M. Kanan \\ University of Belgrade, Faculty of Mathematics, Studentski trg 16, 11000 Beograd, Serbia
}

e-mail: asmaakanan@yahoo.com

Received $12 \mathrm{Feb} 2013$

Accepted 16 Oct 2013

\begin{abstract}
It is known that a square matrix $A$ over a commutative ring $R$ with identity is a left or right zero divisor in $M_{n}(R)$ if and only if the determinant of $A$ is a zero divisor in $R$. Additively inverse commutative semirings with zero 0 and identity 1 are a generalization of commutative rings with identity. In this paper, we present some results for matrices over this type of semiring which generalize the above result for matrices over commutative rings.
\end{abstract}

KEYWORDS: inverse semigroups, positive and negative determinants

\section{INTRODUCTION}

A semiring is an algebraic structure $(S,+, \cdot)$ in which $(S,+, 0)$ and $(S, \cdot, 1)$ are monoids, $1 \neq 0, \cdot$ is distributive over + , and for all $r \in S: r \cdot 0=0 \cdot r=0$. A semiring $(S,+, \cdot)$ is called commutative if it is both additively and multiplicatively commutative.

A semiring $(S,+, \cdot)$ is called additively inverse if $(S,+)$ is an inverse semigroup, i.e., for each $x \in S$ there is a unique $x^{\prime} \in S$ such that $x=x+x^{\prime}+x$ and $x^{\prime}=x^{\prime}+x+x^{\prime}$. An additively inverse commutative semiring with zero and identity is a generalization of a commutative ring with identity. An element $a$ of a ring $R$ is called a left (right) zero divisor if there exists a non-zero $x$ such that $a x=0,(x a=0$ for right zero divisor). We let $M_{n}(R)$ denote the ring of $n \times n$ matrices over $R$.

In this paper, we propose a theorem which generalizes the following theorem: a square matrix $A$ over a commutative ring $R$ with identity is a left or right zero divisor in $M_{n}(R)$ if and only if $\operatorname{det} A \in$ $Z(R)^{1}$, to some semirings in terms of positive and negative determinants.

Some properties of determinants of square matrices over semirings have been studied in Refs. 2, 3 .

In the second section of this paper, we collect some definitions and some propositions about additively inverse semirings. In the third section, we introduce some notions and prove some results for matrices over additively inverse commutative semirings. In the fourth section, we study the zero divisors for square matrices over semirings and prove our main results.

\section{PRELIMINARIES}

In this section, we collect only the necessary notions for the presentation of the main result in the last section.

Definition 1 An element $x$ of a semiring $(S,+, \cdot)$ with zero 0 (identity 1 ) is said to be additively (multiplicatively) invertible if $x+y=y+x=0(x$. $y=y \cdot x=1$ ) for some unique $y \in S$.

Definition 2 A square matrix $A$ over a commutative semiring $S$ is called a left zero divisor in $M_{n}(S)$ if $A B=O$ for some non-zero matrix $B \in M_{n}(S)$. Similarly, $A$ is called a right zero divisor in $M_{n}(S)$ if $C A=O$ for some non-zero matrix $C \in M_{n}(S)$.

Definition $3^{4}$ Let $\mathscr{S}_{n}$ be the symmetric group of degree $n \geqslant 2, \mathscr{A}_{n}$ the alternating group of degree $n$, and $\mathscr{B}_{n}=\mathscr{S}_{n} \backslash \mathscr{A}_{n}$, that is,

$$
\begin{aligned}
& \mathscr{A}_{n}=\left\{\sigma \in \mathscr{S}_{n}: \sigma \text { is an even permutation }\right\}, \\
& \mathscr{B}_{n}=\left\{\sigma \in \mathscr{S}_{n}: \sigma \text { is an odd permutation }\right\} .
\end{aligned}
$$

For $A \in M_{n}(S)$, the positive determinant and the negative determinant of $A$ are defined, respectively, as follows:

$$
\begin{aligned}
& \operatorname{det}^{+} A=\sum_{\sigma \in \mathscr{A}_{n}}\left(\prod_{i=1}^{n} A_{i \sigma(i)}\right), \\
& \operatorname{det}^{-} A=\sum_{\sigma \in \mathscr{B}_{n}}\left(\prod_{i=1}^{n} A_{i \sigma(i)}\right) .
\end{aligned}
$$

We can see that $\mathscr{A}_{n}=\left\{\sigma^{-1}: \sigma \in \mathscr{A}_{n}\right\}$ and $\mathscr{B}_{n}=$ $\left\{\sigma^{-1}: \sigma \in \mathscr{B}_{n}\right\}, \operatorname{det}^{+} I_{n}=1$ and $\operatorname{det}^{-} I_{n}=0$ and for $A \in M_{n}(S), \operatorname{det}^{+} A^{t}=\operatorname{det}^{+} A, \operatorname{det}^{-} A^{t}=\operatorname{det}^{-} A$ (see Ref. 2).

The proof of the following lemma may be found in Ref. 4. 
Lemma 1 For distinct $i, j \in\{1, \ldots, n\}, \sigma \longmapsto \sigma \tau$ is a bijection from $\mathscr{A}_{n}$ onto $\mathscr{B}_{n}$, where $\tau=(i j)$ is a permutation which permutes $i$ and $j$ and leaves the other numbers fixed (a transposition).

We also need the following propositions.

Proposition 1 (Theorem 3 of Ref. 5) If $(S,+, \cdot)$ is an additively inverse semiring, then for all $x, y \in S$,

(i) $\left(x^{\prime}\right)^{\prime}=x$;

(ii) $(x+y)^{\prime}=y^{\prime}+x^{\prime}$;

(iii) $(x y)^{\prime}=x^{\prime} y=x y^{\prime}$;

(iv) $x^{\prime} y^{\prime}=x y$.

Proposition 2 (Ref. 4) If $(S,+, \cdot)$ is an additively inverse semiring with zero 0 and $x, y \in S$ are such that $x+y=0$, then $y=x^{\prime}$.

It is known that a commutative semiring $S$ may be embedded into a ring if and only if it satisfies the additive cancellation law: if $a+x=a+y$, it follows that $x=y$ (see, e.g., Ref. 6).

Example 1 There are additively inverse semirings which cannot be embedded into a ring. For example, let $(S,+, \cdot)=([0,1], \oplus, \odot)$, where $[0,1]=\{x \in$ $\mathbb{R}: 0 \leqslant x \leqslant 1\}, x \oplus y:=\max \{x, y\}$ and $x \odot y:=$ $\min \{x, y\}$. We know that this is an inverse semiring (see, e.g., Ref. 4). However, since $1+1 / 2=1=$ $1+1 / 3$, and $1 / 2 \neq 1 / 3$, this semiring does not satisfy additive cancellation law, so it cannot be embedded into a ring.

\section{AUXILIARY RESULTS}

In the following, all semirings will be commutative inverse semirings with zero 0 and identity 1 . In particular, in $M_{n}(S)$ there is the identity matrix $I_{n}$, all of whose diagonal elements equal to 1 , and all non-diagonal elements equal to 0 . It is clear that $A \cdot I_{n}=I_{n} \cdot A=A$ for every $A \in M_{n}(S)$.

Let $S$ be an additively inverse semiring, $a \in S$ and $n \geqslant 0$, a non-negative integer. We define $a^{(n)}$ as follows:

$$
a^{(n)}:= \begin{cases}a, & \text { if } n \text { is even, } \\ a^{\prime}, & \text { if } n \text { is odd. }\end{cases}
$$

It is easy to check that $\left(a^{(n)}\right)^{(m)}=a^{(n+m)}$ and that $a^{(n)} a^{(m)}=a^{(n+m)}$.

Using this notation, we define the determinant for matrices over additively inverse semirings.

Definition 4 Let $S$ be an additively inverse semiring

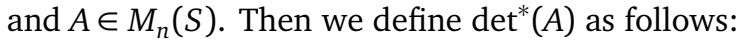

$$
\operatorname{det}^{*}(A):=\operatorname{det}^{+}(A)+\left(\operatorname{det}^{-}(A)\right)^{\prime} .
$$

Note that if we put $\operatorname{sgn}(\sigma)=0$, for even permutations, and $\operatorname{sgn}(\sigma)=1$ for odd permutations, we have that

$$
\operatorname{det}^{*}(A)=\sum_{\sigma \in \mathscr{S}_{n}}\left(\prod_{i=1}^{n} A_{i \sigma(i)}\right)^{\operatorname{sgn}(\sigma)},
$$

which is completely analogous to the usual expansion of the determinant.

We can also define the adjoint matrix of a given matrix in $M_{n}(S)$ as follows.

Definition 5 Let $S$ be an additively inverse semiring and $A \in M_{n}(S)$. For $i, j \in\{1, \ldots, n\}$, let $\mathscr{M}_{i j}(A) \in$ $M_{n-1}(S)$ be a matrix obtained from the matrix $A$ by deleting the $i$ th row and $j$ th column from this matrix. Then $\operatorname{adj}^{*}(A) \in M_{n}(S)$ is defined as

$$
\operatorname{adj}^{*}(A)_{i j}=\left(\operatorname{det}^{*}\left(\mathscr{M}_{j i}(A)\right)\right)^{(i+j)} .
$$

This definition is, as in the case $\operatorname{of}^{\operatorname{det}^{*}}(A)$, completely analogous to the usual definition of the adjoint matrix. It is easy to see that the Laplace expansion with respect to any row holds in our case for det* (the proof is completely analogous to the usual case, so we omit it).

Theorem 1 If $S$ is an additively inverse semiring, $A \in$ $M_{n}(S)$ and $i \in\{1, \ldots, n\}$, one has:

$$
\operatorname{det}^{*}(A)=\sum_{j=1}^{n} A_{i j}\left(\operatorname{det}^{*}\left(\mathscr{M}_{i j}(A)\right)\right)^{(i+j)}
$$

Example 2 Let us assume that $a b$ is additively invertible and let $A \in M_{2}(S)$ be the matrix

$$
A=\left(\begin{array}{ll}
a & b \\
a & b
\end{array}\right) .
$$

Then $\operatorname{det}^{*}(A)=a b+b a^{\prime}=a b+(a b)^{\prime}=0$.

Keeping this example in mind, one should not be surprised that the following theorem holds.

Theorem 2 Let $S$ be an additively inverse semiring and $A \in M_{n}(S)$ be a square matrix such that for all $i, j, k, j \neq k$ the elements $A_{i j} A_{i k}$ are additively invertible. If, in addition to that, $A$ has two equal rows, then $\operatorname{det}^{*}(A)=0$.

Proof: Let us assume that the $r$ th and $s$ th rows are equal, so $A_{r j}=A_{s j}$ for all $j$. Using Lemma 1, with $\tau=(r s)$, we have

$$
\operatorname{det}^{*}(A)=\sum_{\sigma \in \mathscr{A}_{n}} \prod_{i=1}^{n} A_{i \sigma(i)}+\sum_{\sigma \in \mathscr{A}_{n}}\left(\prod_{i=1}^{n} A_{i(\sigma \tau)(i)}\right)^{\prime} .
$$


Since $\tau$ is the transposition ( $r s$ ) we obtain, using results from Proposition 1 and the fact that $A_{r j}=A_{s j}$ for all $j$ :

$$
\begin{gathered}
\operatorname{det}^{*}(A)=\sum_{\sigma \in \mathscr{A}_{n}}\left(A_{r \sigma(r)} A_{s \sigma(s)} \prod_{i \neq r, s} A_{i \sigma(i)}\right) \\
+\sum_{\sigma \in \mathscr{A}_{n}}\left(\left(A_{r \sigma(s)} A_{s \sigma(r)}\right)^{\prime} \prod_{i \neq r, s} A_{i \sigma(i)}\right) \\
=\sum_{\sigma \in \mathscr{A}_{n}}\left(\left(A_{r \sigma(r)} A_{s \sigma(s)}+\left(A_{r \sigma(s)} A_{s \sigma(r)}\right)^{\prime}\right) \prod_{i \neq r, s} A_{i \sigma(i)}\right) \\
=\sum_{\sigma \in \mathscr{A}_{n}}\left(\left(A_{r \sigma(r)} A_{r \sigma(s)}+\left(A_{r \sigma(s)} A_{r \sigma(r)}\right)^{\prime}\right) \prod_{i \neq r, s} A_{i \sigma(i)}\right) \\
=0,
\end{gathered}
$$

since $A_{r \sigma(s)} A_{r \sigma(r)}$ is invertible, so all summands are equal to zero.

Example 3 It is not necessarily the case that the determinant of a matrix with two equal rows is zero. For example, let $(S,+, \cdot)=([0,1], \oplus, \odot)$, the semiring from Example 1. Then for

$$
A=\left(\begin{array}{ll}
1 & 1 \\
1 & 1
\end{array}\right)
$$

we have $\operatorname{det}^{*}(A)=1 \cdot 1+1 \cdot 1^{\prime}=1+1=1$.

The following theorem is vital for our main results.

Theorem 3 Let $S$ be an additively inverse semiring, $A \in M_{n}(S)$ is such that $A_{i j} A_{i k}$ are additively invertible for all $i, j, k$, such that $j \neq k$. Then

$$
A \cdot \operatorname{adj}^{*}(A)=\operatorname{det}^{*}(A) I_{n},
$$

where $I_{n} \in M_{n}(S)$ is the identity matrix.

Proof: The proof is standard. The $(i, k)$ th component of the product is

$$
\begin{gathered}
\left(A \cdot \operatorname{adj}^{*}(A)\right)_{i k}=\sum_{j=1}^{n} A_{i j} \operatorname{adj}^{*}(A)_{j k} \\
=\sum_{j=1}^{n} A_{i j}\left(\operatorname{det}^{*}\left(\mathscr{M}_{k j}(A)\right)\right)^{(k+j)} .
\end{gathered}
$$

If $i=k$, we have

$$
\begin{aligned}
\left(A \cdot \operatorname{adj}^{*}(A)\right)_{i i} & =\sum_{j=1}^{n} A_{i j} \operatorname{adj}^{*}(A)_{j i} \\
= & \sum_{j=1}^{n} A_{i j}\left(\operatorname{det}^{*}\left(\mathscr{M}_{i j}(A)\right)\right)^{(i+j)}=\operatorname{det}^{*}(A) .
\end{aligned}
$$

If $i \neq k$ then we also have an expansion of the determinant of a matrix, but in this case this matrix has equal $i$ th and $k$ th row. Hence from Theorem 2, we conclude that this sum is equal to zero.

Remark 1 One can check that the equality

$$
A \cdot \operatorname{adj}^{*}(A)=\operatorname{det}^{*}(A) I_{n}
$$

need not be true for all matrices $A \in M_{n}(S)$. One can check the same matrix as in Example 3.

\section{MAIN RESULTS}

In this section we prove the main results.

Theorem 4 Let $S$ be an additively inverse semiring with zero 0 and identity 1 and $A \in M_{n}(S)$ is such that $A_{i j} A_{i k}$ is additively invertible for all $i, j, k, j \neq k$. If $A$ is a right zero divisor, then $\operatorname{det}^{*}(A)$ is a zero divisor.

Proof: Since $A$ is a right zero divisor, there exists a non-zero matrix $B \in M_{n}(S)$ such that $B \cdot A=O$. If we multiply this equality on the right by $\operatorname{adj}^{*}(A)$, we obtain

$$
B \cdot A \cdot \operatorname{adj}^{*}(A)=O
$$

and taking into account results from Theorem 3, we obtain

$$
B \operatorname{det}^{*}(A)=O .
$$

Since $B \neq O$, there is a component $B_{i j} \neq 0$, such that $B_{i j} \operatorname{det}^{*}(A)=0$, so $\operatorname{det}^{*}(A)$ is a zero divisor.

Theorem 5 Let $S$ be an additively inverse semiring with zero 0 and identity 1 and $A \in M_{n}(S)$ is such that $A_{i j} A_{i k}$ is additively invertible for all $i, j, k, j \neq k$. If $\operatorname{det}^{*}(A)$ is a zero divisor, then $A$ is a left zero divisor.

Proof: Since $\operatorname{det}^{*}(A)$ is a zero divisor, there exists $x \in S$ such that $\operatorname{det}^{*}(A) \cdot x=0$. If $A_{i j} \cdot x=0$ for all $i, j$, then $A \cdot\left(x I_{n}\right)=O$ and we are done. Hence let us assume that there exist $i, j$ such that $A_{i j} \cdot x \neq 0$. Since $\operatorname{det}^{*}(A) \cdot x=0$, there must exist maximum $r$ such that $1 \leqslant r \leqslant n-1$ and $x$ annihilates all determinants of submatrices of $A$ of order $r+1$, while there exists a submatrix $C$ of order $r$ of $A$ such that $\operatorname{det}^{*}(C) \cdot x \neq 0$. We may assume without loss of generality (and in order to simplify notation) that it is the submatrix formed by the first $r$ rows and columns of matrix $A$. Let us denote by $B$, the submatrix of $A$ formed by the 
first $r+1$ rows and columns of $A$. We claim that

$$
A \cdot\left(\begin{array}{c}
\left(\mathscr{M}_{r+1,1}(B)\right)^{(r+2)} x \\
\left(\mathscr{M}_{r+1,2}(B)\right)^{(r+3)} x \\
\vdots \\
\left(\mathscr{M}_{r+1, r+1}(B)\right)^{(2 r+2)} x \\
0 \\
\vdots \\
0
\end{array}\right)
$$

is zero. If $1 \leqslant i \leqslant r$ we have

$$
\sum_{j=1}^{r+1} A_{i j}\left(\mathscr{M}_{r+1, j}(B)\right)^{(r+1+j)}=0,
$$

since this is just the determinant of a matrix of order $r+1$ having the same $i$ th and $r+1$ st row (we replace $r+1$ st row of matrix $B$ with its $i$ th row).

For $i=r+1$, we have

$$
\sum_{j=1}^{r+1} A_{r+1, j}\left(\mathscr{M}_{r+1, j}(B)\right)^{(r+1+j)} x=\operatorname{det}^{*}(B) x=0,
$$

since $x$ annihilates all determinants of submatrices of $A$ of order larger than $r$. The same conclusion holds for $i>r+1$, since the corresponding sum is just determinant (or determinant') of a submatrix of order larger than $r$ of matrix $A$.

On the other hand, $\left(\mathscr{M}_{r+1, r+1}(B)\right)^{(2 r+2)} x=$ $\operatorname{det}^{*}(C) x \neq 0$ by assumption, so this column is not equal to zero. If we add $n-1$ zero columns to this one, we obtain a matrix $D \neq O$, such that $A \cdot D \neq O$ and we conclude that $A$ is a left zero divisor.

Corollary 1 Let $A \in M_{n}(S)$ be a matrix with entries in an additively inverse semiring $S$, such that $A_{i j} A_{i k}$ is additively invertible for all $i, j, k, j \neq k$. Then, if $A$ is a right zero divisor, then $A$ is a left zero divisor.

Proof: The proof follows directly from previous theorems. Namely, if $A$ is a right zero divisor, then by Theorem $4, \operatorname{det}^{*}(A)$ is a zero divisor, so, by Theorem $5, A$ is a left zero divisor.

Remark 2 The set of left zero divisors in $M_{n}(S)$ may differ from the set of right zero divisors, even if the conditions concerning its components as in previous theorems hold. This is shown in the following example.

Example 4 Let $(S,+, \cdot)=([0,1], \oplus, \odot)$, the semiring from Example 1. The matrix

$$
A=\left(\begin{array}{ll}
1 & 0 \\
1 & 0
\end{array}\right)
$$

is a left zero divisor, but it is not a right zero divisor. Namely,

$$
\left(\begin{array}{ll}
1 & 0 \\
1 & 0
\end{array}\right) \cdot\left(\begin{array}{ll}
0 & 0 \\
1 & 1
\end{array}\right)=\left(\begin{array}{ll}
0 & 0 \\
0 & 0
\end{array}\right)
$$

so $A$ is a left zero divisor. Let us show that $A$ is not a right zero divisor. Suppose that $B \in M_{n}(S)$ is such that $B \cdot A=O$. Hence if

$$
B=\left(\begin{array}{ll}
x & y \\
z & t
\end{array}\right),
$$

we obtain

$$
\left(\begin{array}{ll}
x & y \\
z & t
\end{array}\right) \cdot\left(\begin{array}{ll}
1 & 0 \\
1 & 0
\end{array}\right)=\left(\begin{array}{ll}
0 & 0 \\
0 & 0
\end{array}\right)
$$

It follows that $\max \{x, y\}=x \oplus y=0$ and $\max \{z, t\}=z \oplus t=0$. We conclude that $x=y=$ $z=t$. Hence $B=O$ and $A$ is not a right zero divisor.

Acknowledgements: The author thanks the anonymous referees for their careful reading of the manuscript and useful suggestions.

\section{REFERENCES}

1. Brown WC (1993) Matrices over Commutative Rings, Marcel Dekker, Inc., New York.

2. Ghosh S (1996) Matrices over semirings. Inform Sci 90, 221-30.

3. Reutenauer C, Straubing H (1984) Inversion of matrices over a commutative semiring. J Algebra 88, 350-60.

4. Sombatboriboon S, Mora W, Kemprasit Y (2011) Some results concerning invertible matrices over semirings. Sci Asia 37, 130-5.

5. Karvellas PH (1974) Inversive semirings. J Aust Math Soc 18, 277-88.

6. Hebisch U, Weinert HJ (1998) Semirings-Algebraic Theory and Applications in Computer Science, Series in Algebra vol 5, World Scientific, Singapore. 